\title{
Doctor of Divinity
}

National Cancer Institute

\section{Source}

National Cancer Institute. Doctor of Divinity. NCI Thesaurus. Code C71358.

A doctoral degree in religion. 